\title{
QoS Enhancement by varying Transmission Range in Wireless Ad-hoc Networks
}

\author{
P. Arivubrakan \\ M.E Pervasive Computing Technologies, \\ Anna University of Technology, \\ Tiruchirappalli-620 024 \\ Tamil Nadu-India
}

\author{
V.R. Sarma Dhulipala \\ Assistant Professor (Physics), \\ Centre for Convergence of Technologies, \\ Anna University of Technology, \\ Tiruchirappalli-620 024,Tamil Nadu-India
}

\begin{abstract}
Ad-hoc network is an infrastructureless network which consists of a set of nodes, communicate over a transmission radio. It does not require any central administration. In this paper, we evaluate some of the widely used efficient routing protocols with varying transmission range of the node. Data transmitted by a node is received by all the nodes within its communication range. We focus on the analysis of varying a range of the transmission in terms of distance. The proposed evaluation was made on routing protocols such as AODV and DSR, which are simulated in Network Simulator (ns2) .The performance of these protocols, is analyzed with selected metrics.
\end{abstract}

\section{Keywords}

Ad-hoc networks, AODV, DSR routing protocols, performance evaluation, transmission range.

\section{INTRODUCTION}

Ad-hoc network [1] is a decentralized type of wireless network that utilizes multi-hop relaying and is capable of operating without any fixed infrastructure. It is also called as an infrastructure-less having shared communication channel and it is more suitable for traffic. Ad-hoc networks employed in such a circumstance should be spread and scalable to a large number of nodes. Instead, these networks are formed in an on-demand fashion as soon nodes come sufficiently close to each other. Ad-hoc network eliminates the need for immobile network components, such as routers and base stations, as well as cabling and central administration.

Each node in the network acts as a host and a router. Differences in the radio transmitter and receiver equipment of nodes, such as different transmission ranges, may direct to uni-directional links, which could complicate routing in the ad-hoc network. The nodes in wireless networks [2] are generally power constrained since they depend on limited battery resources while wireless communications consume more energy. Wireless environment requires more energy consumption due to neighborhood transmissions. Nodes are spending their batteries not only by sending their own packets, but by just overhearing packets from other nodes in the network. As ad-hoc network is based on multi-hop communication, more energy is also spent by forwarding packets to the nodes.

When transmitting nodes are not in the range of each other in wireless ad-hoc networks, they need to rely on multi-hop communications. Under such a constraint, packet forwarding or routing, becomes mandatory. The impact of the radio transmission range affects the network topology and energy consumption significantly [3]. A higher transmission range increases the distance progress of data packets toward their final destinations with less packet drop, and it is achieved at the expense of high-energy consumption per transmission. On the other hand, a shorter transmission range uses less energy to forward packets to the next hop, although a larger number of hops is needed for packets to reach their destinations. By changing the transmission range of nodes, interference can be reduced at the same time as nodes save valuable energy [4].

All nodes, including the source node and the intermediate nodes, employ a common transmission range. Transmission range is directly proportional to the node distance. Therefore, direct transmission occurs only when the destination node is within a range from the source node. Any node within a range of a node is called its neighbor node. We assume that each node knows the locations of all its neighbors and the location of the destination node. A smaller transmission range requires a number of nodes than the high range transmission, and it requires multi hop transmission. The source node transmits a packet to the destination node directly, if the destination node is located within a range from the source node. When the destination node is outside the transmission range of the source node, the source node sends the packet to a neighbor, the neighbor is closer in distance, forward the packet until it reaches to the destination node.

\section{RELATED WORK}

At present, there are several papers related to performance evaluation of Ad-hoc On demand Distance Vector(AODV) and Dynamic Source Routing (DSR) [5,6,7]. We have observed that, though there is a significant difference between AODV and DSR protocol $[8,9,10,11]$ but by varying a transmission range in between them is not yet analyzed and obtain high throughput. So we have focused on the performance of the protocol by changing a communication range and compute the performance metrics such as packet delivery ratio, average end to end delay, throughput and packet loss $[12,13,14]$. In this paper, we observed and analyzed our experiment with the varying transmission range for AODV and DSR in wireless ad-hoc networks.

In the ad-hoc networks, intermittent flooding of routing information is quite expensive, since all nodes compete for access to the wireless medium with limited bandwidth. Special routing protocols are needed for ad-hoc networks. Adhoc routing protocols is to discover a path to be followed by packets from a source node to a destination node, various routing protocols have been proposed. We have selected two, on demand routing protocols such as AODV and DSR for the analysis of transmission range. The advantage of using on 
demand reactive routing protocols such as AODV and DSR avoid the redundant exchange of route information, and it consumes more energy.

\subsection{AODV}

Ad-hoc On demand Distance Vector (AODV) routing protocol [15] is a reactive routing protocol that recognized only when it is required by the source node for transmitting data packets. In AODV, the source node and the neighbor nodes store the hop information related to each flow for data packet transmission. It uses a destination sequence number to determine up to date path information to the destination and avoid the count-to-infinity problem. Source node broadcasts the RREQ (Route Request) to the destination node along with the source node address, destination node address and sequence number. If the node is within the range of the network, the destination node sends the RREP (Route Reply) to the source node. If it is out of range, it sends the RREQ to the neighbor and to the closest neighbor of the destination. It maintains routing table, one entry per destination and an entry is discarded if it is not used recently. If the node is external to the range of the network, it sends the RRER (Route Error) message about the link failure. It is an efficient routing protocol for varying a range of the communication under CBR (Constant Bit Rate) connection. The benefit of the protocol is setup delay is less.

\subsection{DSR}

Dynamic Source Routing (DSR) protocol [16] is a reactive, on demand routing protocol based on the source routing. The usage of source routing irrelevantly allows routing of packets to be loop-free, avoids the need for up-to-date routing information in between nodes and allows nodes that overhear packets containing routes to cache this information for their own future use. A node that needs to send a packet to a destination checks in its route cache if it has a route available. In a route discovery phase, a node sends the route request to the destination node. It responds by the route reply than a path is established between the source and destination. Route maintenance indicates that the source route is broken, it can prefer another route to reach the destination. The advantage of using DSR is that it is beacon-less and hence does not require periodic packet transmission and it performs well in the static network, in between nodes utilize the route cache information efficiently to reduce the control overhead.

AODV and DSR have some differences. In AODV, a node sends a packet to the destination than data packets only contain the destination address. On the other hand,in DSR a node sends a packet to the destination the entire routing information is carried by packets, which cause more routing overhead than AODV.

\section{SIMULATION RESULT}

The performance of AODV \& DSR has been analyzed with varying transmission range with a distance of $50 \mathrm{~m}, 75 \mathrm{~m}$, $100 \mathrm{~m}, 125 \mathrm{~m}$ and $150 \mathrm{~m}$ under CBR connection of simulation time at $3 \mathrm{~ms}$ and $5 \mathrm{~ms}$ using ns $2[17,18]$. We compute the performance of reactive routing protocols with selected metrics such as packet delivery ratio, average end to end delay, packet loss and throughput for a various range.

\subsection{Simulation Parameter}

Table 1: Simulation Parameter

\begin{tabular}{|l|l|}
\hline \multicolumn{1}{|c|}{ Parameter } & \multicolumn{1}{c|}{ Value } \\
\hline Network Simulator & Ns2.29 \\
\hline Channel type & Wireless channel \\
\hline Radio-propagation model & Two Ray Ground \\
\hline Antenna type & Omni Antenna \\
\hline Interface queue type & Drop Tail/PriQueue \\
\hline Routing protocols & AODV/DSR \\
\hline MAC type & $802 \_11$ \\
\hline Transmission range & $50,75,100,125,150$ \\
\hline Traffic Type & CBR \\
\hline Max packet in Queue & 50 \\
\hline Simulation Time & $3 \mathrm{~ms}, 5 \mathrm{~ms}$ \\
\hline
\end{tabular}

\subsection{Parameter Analysis}

For network simulation in ns2, there is several metrics, which are used to evaluate the performance of the routing protocols such as AODV and DSR in wireless ad-hoc networks. Performance metrics are Packet Delivery Ratio, average End to End delay, Packet loss and Throughput.

\subsubsection{Packet Delivery Ratio}

The Packet Delivery Ratio is defined as the ratio between the number of receiving packets of the CBR sink by the number of send packets of the CBR source. The performance is enhanced when the packet delivery ratio is high. The following graphs show the packet delivery ratio provided by the two different on-demand protocols with CBR connections of various transmission ranges.

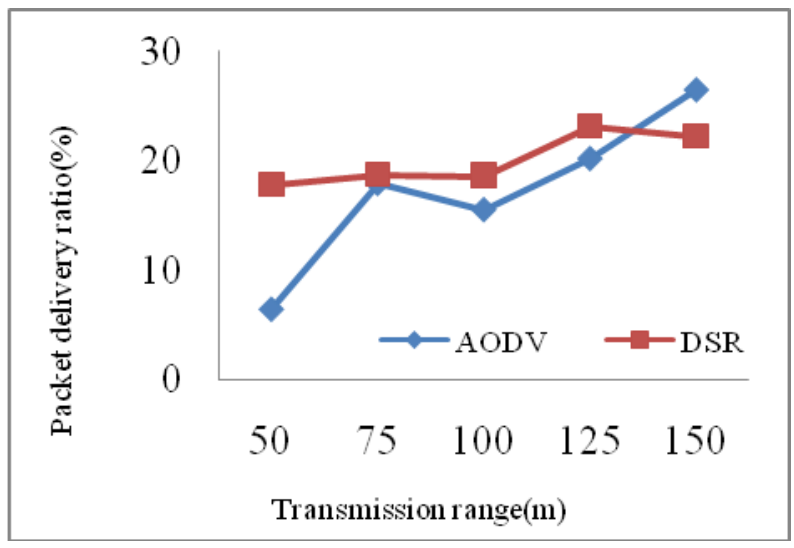

Figure 1 Packet Delivery Ratio at 3ms 


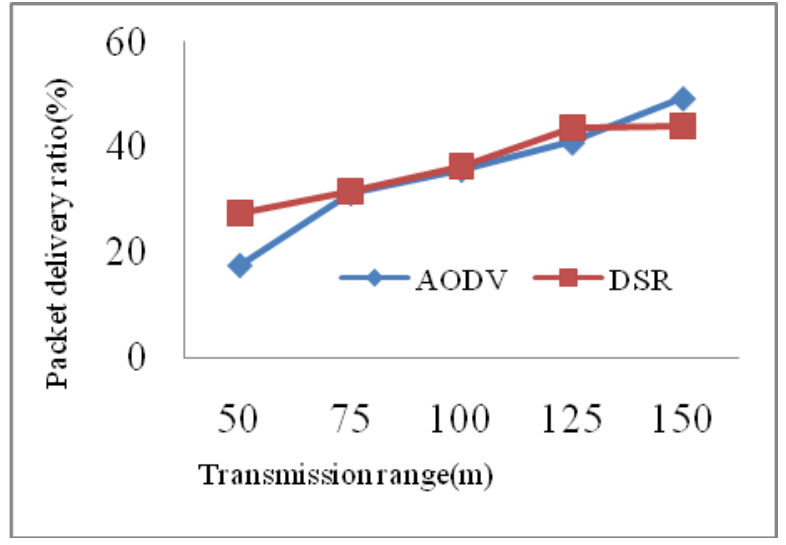

Figure 2 Packet Delivery Ratio at $5 \mathrm{~ms}$

\subsubsection{End to End delay}

Average End-to-End delay is the difference of packet send and received time by the total time difference of the total number of packet received gave the average end-to-end delay for the received packets. The performance is improved when a packet end-to-end delay is low. The following graphs show the end to end delay provided by the two different protocols with CBR connection of various transmission ranges at $3 \mathrm{~ms}$ and $5 \mathrm{~ms}$ simulation time. Average end to end delay of DSR is very low at $125 \mathrm{~m}$ compared to AODV routing protocols.

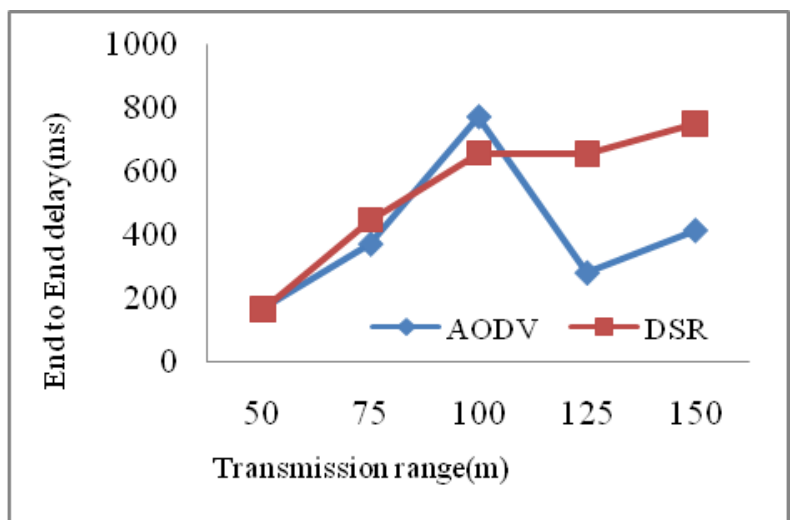

Figure 3 End to End delay at $3 \mathrm{~ms}$

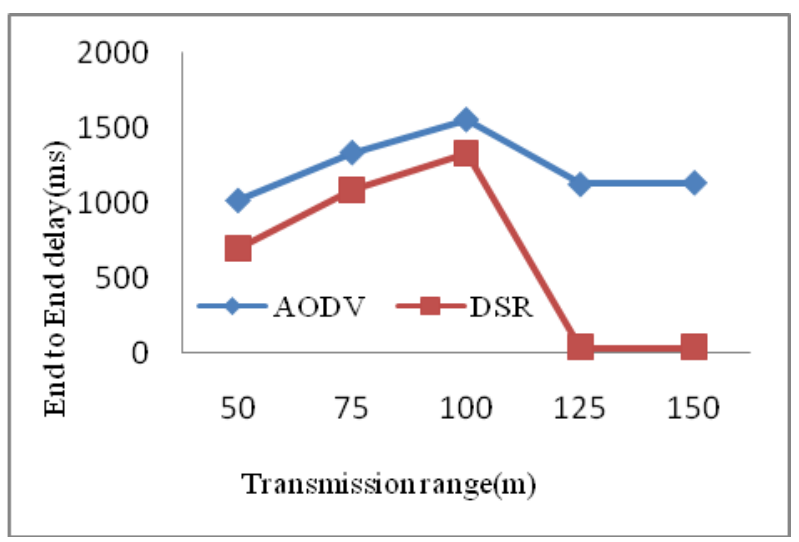

Figure 4 End to End delay at $5 \mathrm{~ms}$

\subsubsection{Packet loss}

Packet loss is calculated by the number of packets that never reached the destination. The following graphs show packet loss provided by the two different protocols with CBR connection of various transmission ranges at $3 \mathrm{~ms}$ and $5 \mathrm{~ms}$.Packet loss is less at larger transmission range than the shorter transmission range of simulation time $5 \mathrm{~ms}$.

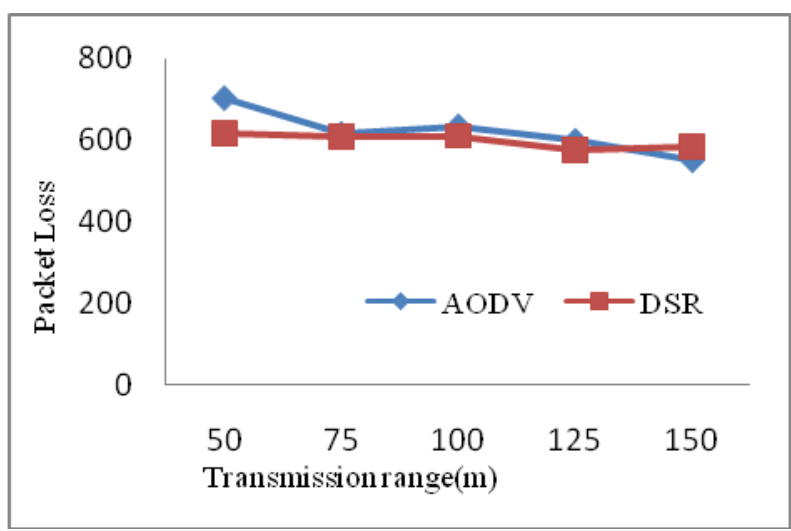

Figure 5 Packet loss at 3ms

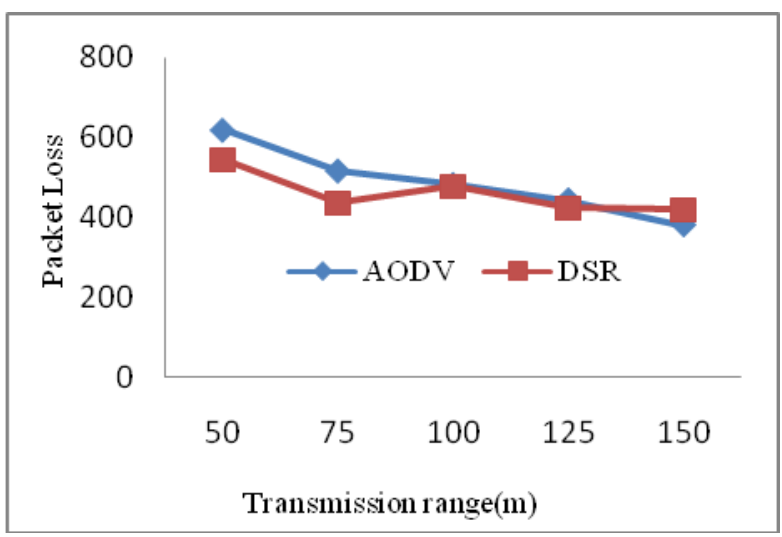

Figure 6 Packet loss at $5 \mathrm{~ms}$

\subsubsection{Throughput}

Throughput defines the number of packets arriving at the destination per second. The following graphs show the throughput provided by the two different reactive protocols with CBR connection of various transmission ranges at $3 \mathrm{~ms}$ and $5 \mathrm{~ms}$ simulation time. Maximum simulation time of DSR gave the highest throughput in $125 \mathrm{~m}$ range compared to AODV routing protocol. As shown in the graph, DSR performed well in terms of throughput compared to AODV. 


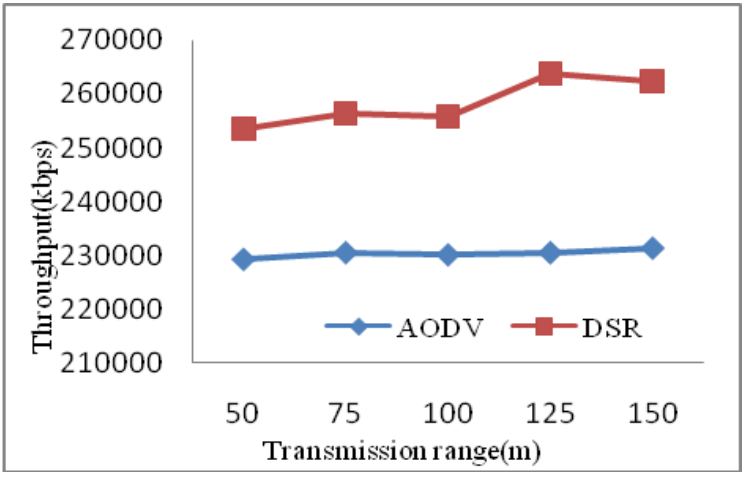

Figure 7 Throughput at 3ms

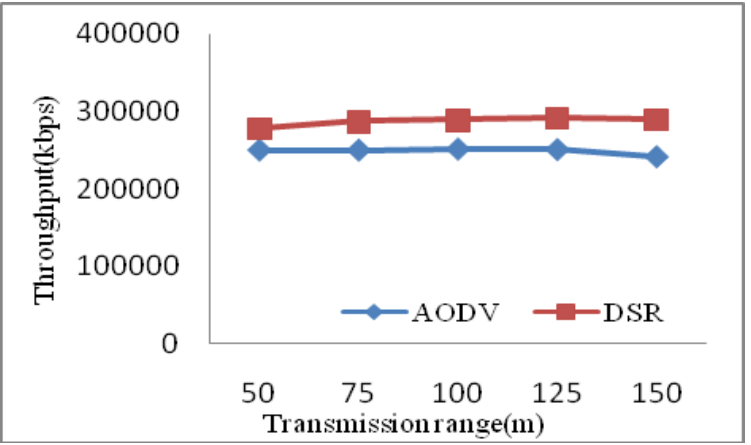

Figure 8 Throughput at $3 \mathrm{~ms}$

\section{CONCLUSION}

The transmission range as a system parameter affects the overall energy consumption of wireless ad-hoc networks. The performance of these two reactive routing protocols shows some differences by varying transmission range and simulation time. From our experimental analysis we conclude that DSR has maximum throughput, high packet delivery ratio, loss of packet is less and end to end delay is low compared to the AODV routing protocol at $5 \mathrm{~ms}$. We compare the two protocols in the analyzed scenario, we found that overall performance of DSR is better than AODV routing protocols at $125 \mathrm{~m}$ range. The performance enhanced in higher transmission range. Our results can be used to determine the proper radio transmission range for the reactive routing protocols such as AODV and DSR in wireless ad-hoc networks without degrading a system performance.

\section{REFERENCES}

[1] Siva Ram Murthy, C., and Manoj, B.S. Second Edition, Low price Edition, Pearson Education, 2007.Ad-hoc Wireless Networks, Architectures and Protocols.

[2] Feeney, L.M., and Nilsson, M., "Investigating the energy consumption of a wireless network interface in an Adhoc networking environment", In Proceedings of the IEEE INFOCOM, pp. 1548 - 1557, 2001.

[3] Ramanathan, R., and Rosales-Hain, R.,"Topology control of multihop wireless networks using transmit power adjustment", In Proceedings of the IEEE INFOCOM, pp. 404-413, 2000.

[4] Suchismita Chinara and Santanu Kumar Rath, "Topology Control by Transmission Range Adjustment Protocol for Clustered Mobile Ad Hoc Networks",ISRN Communications and Networking,2011
[5] Usop, N., Abdullah, A., and Abidin, A., "Performance Evaluation of AODV, DSDV and DSR routing Protocol in Grid Environment", International Journal of Computer and Network Security, vol. 9, no. 7, 2009.

[6] Rahman, A., and Zukarnain, Z., "Performance Comparison of AODV, DSDV and I-DSDV Routing Protocols in Mobile Ad-hoc Networks", European Journal of Scientific Research, vol. 31, no. 4, pp. 566576, 2009.

[7] Harminder S. Bindra, Sunil K. Maakar and Sangal, A.L., " Performance Evaluation of Two Reactive Routing Protocols of MANET using Group Mobility Model",IJCSI International Journal of Computer Science Issues, vol. 7, no. 10, May 2010.

[8] Anwar, F., Azad, M., Rahman,M., and Uddin,M., "Performance Analysis of Ad-hoc Routing Protocols in Mobile WiMAX Environment,"IAENG International Journal of Computer Science, vol. 35, no. 3, pp. 353359,2008

[9] Vivek Thaper, Bindyia Jain, and Varsha Sahni, "Performance analysis of adhoc routing protocols using random waypoint mobility model in wireless sensor networks", International Journal on Computer Science and Engineering (IJCSE), August 2011.

[10] Sreerama Murty, M.,and Venkat Das,M., "Performance Evalution of MANET Routing Protocols using Reference Point Group Mobility and Random WayPoint Models",International Journal of Ad hoc, Sensor \& Ubiquitous Computing (IJASUC) vol.2, no.1, March 2011

[11] Nadesh, R. K., Sumathy, D., and Benjula Anbu Malar, M.B. "Performance Analysis of MANET (WLAN) Using Different Routing Protocols in Multi service Environments-An Quantitative Study", Advanced Networking and Applications vol. 03, no.02, pp:10761079,2011 .

[12] Boomarani Malany,A.,Sarma Dhulipala, V.R., and Chandrasekaran, RM , "Throughput and Delay Comparison of MANET Routing Protocols" ,Int. J. Open Problems Compt. Math.,vol. 2, no. 3, September 2009.

[13] Rajneesh Kumar Gujral, and Manpreet Singh, "Performance Analysis of Ad hoc Routing Protocols for Voice Communication Support over Hybrid MANETs", IJCA vol. 22,no.3, May 2011.

[14] Godder,T.K., Hossain,M.M., Rahman,M.M., Sipon Miah,Md and Mondal, S. K., "Performance comparison and analysis of mobile ad hoc routing Protocols" ,Computer Science \& Engineering: An International Journal (CSEIJ), vol.1, no.1, April 2011.

[15] Charles Perkins, Elizabeth Royer, and Samir Das., "Adhoc on demand distance vector (AODV) routing", July 2003.

[16] David B. Johnson and David A. Maltz, "Dynamic source routing in Ad-hoc wireless networks", Technical report, Carnegie Mellon University, 1996.

[17] NETWORK SIMULATOR (ns2). http://www.isi.edu/nsnam/ns/.

[18] Marc Geris' Tutorial for the UCB/LBNL/VINT Network Simulator "ns" 